\title{
Conference reflections
}

\author{
Technology breakthroughs at the 2019 IEEE International Electron Devices Meeting.
}

or a long time, conferences have
played a key role in the discussion
and development of research. And though the climate change crisis now demands reflection on the necessity of any event - and, in particular, the necessity of any air travel attending might require $^{1}$ - they remain central to various research communities across science and engineering. For work on semiconductor and electronic device technology, the IEEE International Electron Devices Meeting (IEDM), which takes place in San Francisco in December, is one such event.

Last year, we highlighted some of the breakthroughs reported at the 2018 meeting. We also took the opportunity to introduce our policy on conference proceedings. (To reiterate, we are happy to consider work containing material that has been published previously in a conference proceedings paper; the submission should though provide a substantial extension in terms of results, methodology, analysis, conclusions and/or implications over the proceedings paper.) In this issue of Nature Electronics, we return to IEDM and offer our highlights of the 2019 meeting.

In our coverage of IEDM 2018, we showcased ${ }^{2}$ a report from Intel on integrating magnetoresistive random access memory (MRAM) with their 22-nm fin field-effect transistor (FinFET) technology ${ }^{3}$. (Similar developments were also reported by Samsung at the event ${ }^{4}$.) MRAM is a non-volatile memory technology in which information is stored in elements known as magnetic tunnel junctions; originally, switching in these junctions was achieved using a magnetic field, but it can now be done electrically via an effect known as spin-transfer torque (STT). At IEDM 2019, Intel now describe a two-megabyte STT-MRAM array that meets the specification for level-4 cache application, work Yizhou Liu and Guoqiang Yu of Beijing National Laboratory for Condensed Matter Physics discuss in a News \& Views article.

Also at IEDM 2019, researchers at the Taiwan Semiconductor Manufacturing Company (TSMC) detail their 5-nm complementary metal-oxide-semiconductor (CMOS) technology, work Toshiro Hiramoto of the University of Tokyo explores in a News \& Views article. The technology is intended for use in both mobile and high-performance computing applications, despite their seemingly contrasting requirements, and compared to the company's previous 7-nm technology, it offers an improved logic density of around 1.84 times, and a gain in speed of $15 \%$ or a power reduction of $30 \%$.

IEDM is a mix of academia and industry, an important combination for the development of new and innovative technologies. And it is collaborative work from researchers in both arenas that features in our third highlighted article. In particular, Jun Ma, Elison Matioli and colleagues at the École Polytechnique Fédérale de Lausanne and Enkris Semiconductor report on multichannel $\mathrm{AlGaN} / \mathrm{GaN}$ power transistors, a result Martin Kuball of the University of Bristol discusses in a News \& Views article.
By using a slanted tri-gate geometry, the device can offer both a high breakdown voltage of $1,200 \mathrm{~V}$ and low on-resistance of $2.8 \Omega \mathrm{mm}$.

Elsewhere we highlight work from Sami Oukassi and colleagues at the University of Grenoble Alpes, CEA-Leti on the development of thin-film batteries with high energy density storage. The batteries, which have thickness of only of $95 \mu \mathrm{m}$, could be of use in implantable medical devices. Also with implications for medical applications, we highlight work from Hidekuni Takao and colleagues at Kagawa University and Takamatsu Red Cross Hospital on the creation of a slip sensor that can visualize the grasp of forceps used by surgeons during keyhole surgery. Finally, we highlight work from Amit Lal and colleagues at Cornell University and $A^{\star}$ STAR, Singapore on integrating aluminium nitride thin-film transducers with 180-nm CMOS technology. The approach provides CMOS-driven gigahertz ultrasonic transducers that the researchers used to measure electrolyte concentrations in solution and to image fingerprints.

Published online: 16 December 2019 https://doi.org/10.1038/s41928-019-0349-4

\footnotetext{
References

1. Nat. Nanotechnol. 14, 813 (2019).

. Raychowdhury, A. Nat. Electron. 1, 618-619 (2018)

3. Golonzka, O. et al. In Proc. 2018 IEEE Int. Electron Devices Meeting 18.1.1-18.1.4 https://doi.org/10.1109/ IEDM.2018.8614620 (2018).

4. Song, Y. J. et al. In Proc. 2018 IEEE Int. Electron Devices Meeting 18.2.1-18.2.4 https://doi.org/10.1109/IEDM.2018.8614635 (2018).
}

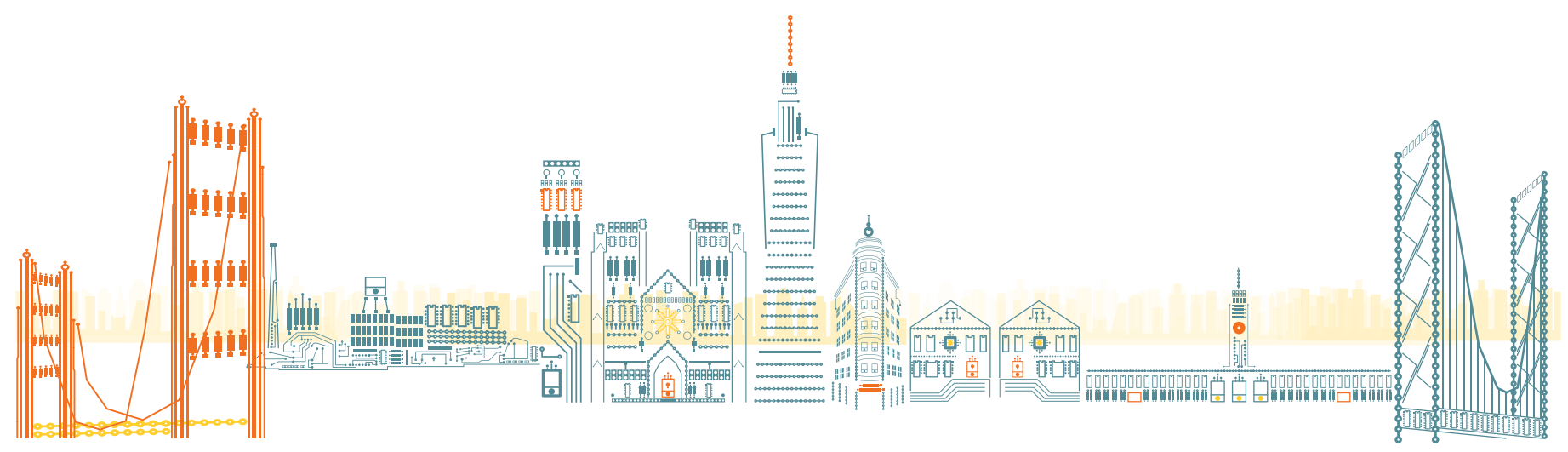

